
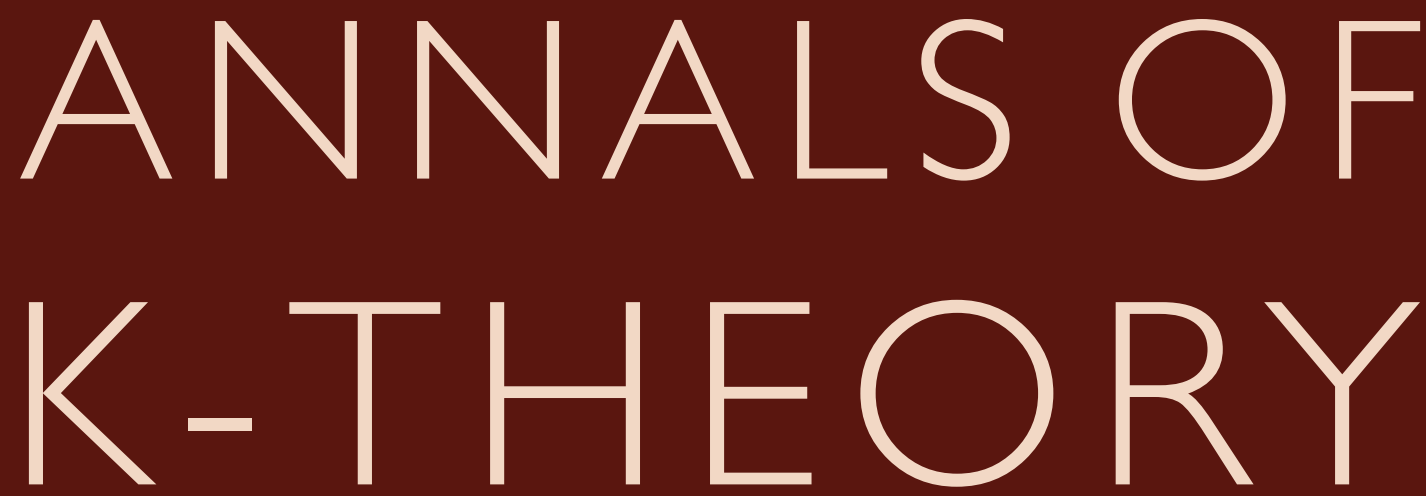

no. 3 vol 12016

The Balmer spectrum of a tame stack

Jack Hall

Thsp

A JOURNAL OF THE K-THEORY FOUNDATION 


\title{
The Balmer spectrum of a tame stack
}

\author{
Jack Hall
}

Let $X$ be a quasicompact algebraic stack with quasifinite and separated diagonal. We classify the thick $\otimes$-ideals of $\mathrm{D}_{\mathrm{qc}}(X)^{c}$. If $X$ is tame, then we also compute the Balmer spectrum of the $\otimes$-triangulated category of perfect complexes on $X$. In addition, if $X$ admits a coarse space $X_{\mathrm{cs}}$, then we prove that the Balmer spectra of $X$ and $X_{\mathrm{cs}}$ are naturally isomorphic.

\section{Introduction}

Let $X$ be a quasicompact and quasiseparated scheme. Let $\operatorname{Perf}(X)$ be the $\otimes$-triangulated category of perfect complexes on $X$. A celebrated result of Thomason [1997, Theorem 3.15], extending the work of Hopkins [1987, Section 4] and Neeman [1992a, Theorem 1.5], is a classification of the thick $\otimes$-ideals of $\operatorname{Perf}(X)$ in terms of the Thomason subsets of $|X|$, which are those subsets $Y \subseteq|X|$ expressible as a union $\cup_{\alpha} Y_{\alpha}$ such that $|X| \backslash Y_{\alpha}$ is quasicompact and open.

If $X$ is a quasicompact and quasiseparated algebraic space, Deligne-Mumford stack, or algebraic stack, then it is also natural to consider the $\otimes$-triangulated category Perf $(X)$ of perfect complexes on $X$ (see [Hall and Rydh 2014, Section 4] for precise definitions).

In general, Thomason's classification of thick $\otimes$-ideals of $\operatorname{Perf}(X)$ fails for algebraic stacks (Example 3.2). If one instead works with the $\otimes$-ideal $\mathrm{D}_{\mathrm{qc}}(X)^{c} \subseteq \operatorname{Perf}(X)$ of compact perfect complexes, then the first main result of this article is that the classification goes through without change.

Theorem 1.1 (classification of thick $\otimes$-ideals). If $X$ is a quasicompact algebraic stack with quasifinite and separated diagonal, then there is a bijective and inclusion preserving correspondence between the thick $\otimes$-ideals of $\mathrm{D}_{\mathrm{qc}}(X)^{c}$ and the Thomason subsets of $|X|$.

Some special cases of Theorem 1.1 are the following:

- If $k$ is a field and $G$ is a finite group, then $\mathrm{D}^{b}(\operatorname{Proj} k G)$ has no nontrivial $\otimes$-ideals.

MSC2010: primary 14F05; secondary 13D09, 14A20, 18G10.

Keywords: derived categories, algebraic stacks. 
- If $Y$ is a quasiprojective scheme over a field $k$ with a proper action of an affine group scheme $G$, then the thick $\otimes$-ideals of $\mathrm{D}\left(\mathrm{Q} \operatorname{Coh}^{G}(Y)\right)^{c}$ are in bijective correspondence with the $G$-invariant Thomason subsets of $X$.

The first special case is easy to prove directly and is well-known (for example, [Benson et al. 2011, Proposition 2.1]). In some sense, this makes our results orthogonal to those of [Benson et al. 2011]. The second special case was only known in characteristic 0 when $Y$ was normal or quasi-affine [Krishna 2009, Theorem 7.8] or in characteristic $p$ when $G$ is finite of order prime to $p$ and $X$ is smooth [Dubey and Mallick 2012, Theorem 1.2].

We prove Theorem 1.1 using tensor nilpotence with parameters (Theorem 2.3), which extends [Thomason 1997, Theorem 3.8] and [Hopkins 1987, Theorem 10ii] (compare [Neeman 1992a, 1.1]) to quasicompact algebraic stacks with quasifinite and separated diagonal. As should be expected, stacks of the form $[Y / G]$, where $Y$ is an affine variety over a field $k$ and $G$ is a finite group with order divisible by the characteristic of $k$, are the most troublesome. This is dealt with in Lemma 2.6, which relies on some results developed in Appendix A.

If $\mathcal{T}$ is a $\otimes$-triangulated category, then Balmer [2005] has functorially constructed from $\mathscr{T}$ a locally ringed space $\operatorname{Sp}_{\mathrm{Bal}}(\mathscr{T})$, the Balmer spectrum. A fundamental result of Balmer [2005, Theorem 5.5], which was extended by Buan, Krause and Solberg [Buan et al. 2007, Theorem 9.5] to the non-noetherian setting, is that if $X$ is a quasicompact and quasiseparated scheme, then there is a naturally induced isomorphism

$$
X \rightarrow \operatorname{Sp}_{\mathrm{Bal}}(\operatorname{Perf}(X))
$$

An algebraic stack is tame if its stabilizer groups at geometric points are finite linearly reductive group schemes [Abramovich et al. 2008, Definition 2.2]. Every scheme and algebraic space is tame. Moreover, in characteristic zero, a stack is Deligne-Mumford if and only if is tame. In characteristic $p>0$, there are nontame Deligne-Mumford stacks (e.g., $\left.B_{\mathbb{F}_{p}}(\mathbb{Z} / p \mathbb{Z})\right)$ and tame stacks that are not DeligneMumford (e.g., $B_{\mathbb{F}_{p}} \mu_{p}$ ). Nagata's theorem [Hall and Rydh 2015, Theorem 1.2] provides a classification of finite linearly reductive group schemes over fields, which allows one to determine whether a given algebraic stack is tame. Our definition of tame stack is substantially weaker than that what appears in [Abramovich et al. 2008, Definition. 3.1] (see Appendix A).

Tame stacks are precisely those stacks with quasifinite diagonal such that the compact objects of $\mathrm{D}_{\mathrm{qc}}(X)$ coincide with the perfect complexes. In particular, for tame stacks $\mathrm{D}_{\mathrm{qc}}(X)^{c}$ contains a monoidal unit and so becomes a $\otimes$-triangulated category. Using Theorem 1.1, we extend the result of [Buan et al. 2007] to tame stacks. 
Theorem 1.2. Let $X$ be a quasicompact algebraic stack with quasifinite and separated diagonal. If $X$ is tame, then there is a natural isomorphism of locally ringed spaces:

$$
\left(|X|, \mathrm{O}_{X_{\mathrm{Zar}}}\right) \rightarrow \operatorname{Sp}_{\mathrm{Bal}}(\operatorname{Perf}(X)),
$$

where $\mathcal{O}_{X_{\text {Zar }}}$ is the Zariski sheaf $U \mapsto \Gamma\left(U, \mathcal{O}_{X}\right)$.

Theorem 1.2 implies that the Balmer spectrum cannot be used to reconstruct locally separated algebraic spaces [Knutson 1971, Example 2]. Balmer [2013] has recently initiated the study of unramified monoids in $\otimes$-triangulated categories and Neeman [2015] has classified them in the case of a separated noetherian scheme. It is hoped that a refinement of the Balmer spectrum can be constructed from unramified monoids, which would — at least - permit the reconstruction of algebraic spaces.

If $X$ is an algebraic stack with finite inertia (e.g., a separated Deligne-Mumford stack), then $X$ admits a coarse space $\pi: X \rightarrow X_{\mathrm{cs}}$ [Keel and Mori 1997; Rydh 2013], which is the universal map from $X$ to an algebraic space. If $X$ has finite inertia, then $X$ has separated diagonal. Thus we can also establish the following.

Theorem 1.3. Let $X$ be a quasicompact, quasiseparated algebraic stack with finite inertia and coarse space $\pi: X \rightarrow X_{\mathrm{cs}}$. If $X$ is tame, then

$$
\operatorname{Sp}_{\mathrm{Bal}}\left(\mathrm{L} \pi^{*}\right): \operatorname{Sp}_{\mathrm{Bal}}(\operatorname{Perf}(X)) \rightarrow \operatorname{Sp}_{\mathrm{Bal}}\left(\operatorname{Perf}\left(X_{\mathrm{cs}}\right)\right)
$$

is an isomorphism of ringed spaces.

Krishna [2009, Theorem 7.10] proved Theorem 1.3 when $X$ is of the form $[W / G]$, where $W$ is quasiprojective and normal or quasi-affine, and $G$ is a linear algebraic group in characteristic 0 acting properly on $W$. Dubey and Mallick [2012, Theorem 1.2] proved a similar result in positive characteristic, but required $W$ to be smooth and $G$ a finite group with order not divisible by the characteristic of the ground field. In particular, Theorem 1.3 is stronger than all existing results and Theorems 1.1 and 1.2 are new.

Assumptions and conventions. A priori, we make no separation assumptions on our algebraic stacks. However, all stacks used in this article will be, at the least, quasicompact and quasiseparated. Usually, they will also have separated diagonal. If $X$ is an algebraic stack, then let $|X|$ denote its associated Zariski topological space [Laumon and Moret-Bailly 2000, Section 5]. For derived categories of algebraic stacks, we use the conventions and notations of [Hall and Rydh 2014, Section 1]. In particular, if $X$ is an algebraic stack, then $\operatorname{Mod}(X)$ is the abelian category of $\mathrm{O}_{X^{-}}$ modules on the lisse-étale site of $X$ and $\mathrm{D}_{\mathrm{qc}}(X)$ denotes the unbounded derived category of $\mathrm{O}_{X}$-modules with quasicoherent cohomology sheaves. If $f: X \rightarrow Y$ is 
a morphism of algebraic stacks, then there is always an adjoint pair of unbounded derived functors

$$
\mathrm{D}_{\mathrm{qc}}(X) \underset{\mathrm{L} f_{\mathrm{qc}}^{*}}{\stackrel{\mathrm{R}\left(f_{\mathrm{qc}}\right)_{*}}{\rightleftarrows}} \mathrm{D}_{\mathrm{qc}}(Y) .
$$

If $f$ is quasicompact, quasiseparated and representable, then $\mathrm{R}\left(f_{\mathrm{qc}}\right)_{*}$ agrees with $\mathrm{R} f_{*}$, the unbounded derived functor of $f_{*}: \operatorname{Mod}(X) \rightarrow \operatorname{Mod}(Y)$ [Hall and Rydh 2014, Lemma 2.5(3) and Theorem 2.6(2)]. If $f$ is smooth, then $\mathrm{L} f_{\mathrm{qc}}^{*}$ agrees with the unique extension of the exact functor $f^{*}: \operatorname{Mod}(Y) \rightarrow \operatorname{Mod}(X)$ to the unbounded derived category.

\section{Tensor nilpotence with parameters}

Definition 2.1. Let $X$ be an algebraic stack and let $\xi: M \rightarrow N$ be a morphism in $\mathrm{D}_{\mathrm{qc}}(X)$. Let $Z \subseteq|X|$ be a subset. We say that $\xi$ vanishes at the points of $Z$ if for every algebraically closed field $k$ and morphism $z: \operatorname{Spec} k \rightarrow X$ that factors through $Z$, then $L z_{\mathrm{qc}}^{*} \xi$ is the zero map in $\mathrm{D}_{\mathrm{qc}}(\operatorname{Spec} k)$.

This definition is connected to a more familiar notion for schemes.

Lemma 2.2. Let $X$ be a scheme and let $\xi: M \rightarrow N$ be a morphism in $\mathrm{D}_{\mathrm{qc}}(X)$. If $Z \subseteq|X|$ is a subset, then $\xi$ vanishes at the points of $Z$ if and only if $\xi \otimes_{\hat{O}_{X}}^{\mathrm{L}} \kappa(z)$ is the zero map in $\mathrm{D}(\kappa(z))$ for every $z \in Z$, where $\kappa(z)$ denotes the residue field of $z$.

Proof. We immediately reduce to the situation where $X=\operatorname{Spec} \kappa$ and $\kappa$ is a field. It now suffices to prove that if $\kappa \subseteq k$ is a field extension, where $k$ is algebraically closed, then $\xi \otimes k$ is the zero map in $\mathrm{D}(k)$ if and only if $\xi$ is the zero map in $\mathrm{D}(\kappa)$. This is obvious.

If $K \in \mathrm{D}_{\mathrm{qc}}(X)$, then the cohomological support of $K$ is defined to be the subset

$$
\operatorname{supph}(K)=\bigcup_{n \in \mathbb{Z}} \operatorname{supp}\left(\mathscr{H}^{n}(K)\right) \subseteq|X| .
$$

For the basic properties of cohomological support, see [Hall and Rydh 2014, Lemma 4.8], which extends [Thomason 1997, Lemma 3.3] to algebraic stacks. The main result of this section is the following theorem.

Theorem 2.3 (tensor nilpotence with parameters). Let $X$ be a quasicompact algebraic stack with quasifinite and separated diagonal. Let $\psi: E \rightarrow F$ be a morphism in $\mathrm{D}_{\mathrm{qc}}(X)$, where $E \in \mathrm{D}_{\mathrm{qc}}(X)^{c}$. Let $K \in \operatorname{Perf}(X)$. If $\psi$ vanishes at the points of $\operatorname{supph}(K)$, then there exists a positive integer $n$ such that $K \otimes_{\mathfrak{O}_{X}}^{\mathrm{L}}\left(\psi^{\otimes n}\right)=0$ in $\mathrm{D}_{\mathrm{qc}}(X)$.

The following example demonstrates that Theorem 2.3 cannot be weakened to the situation where $E \in \operatorname{Perf}(X)$. 
Example 2.4. Let $X=B_{\mathbb{F}_{2}}(\mathbb{Z} / 2 \mathbb{Z})$, which is a quasicompact, nontame DeligneMumford stack with finite diagonal. Consider the adjunction morphism

$$
\eta: \mathrm{O}_{X} \rightarrow x_{*} \mathrm{O}_{\mathbb{F}_{2}}
$$

where $x: \operatorname{Spec} \mathbb{F}_{2} \rightarrow X$ is the usual cover. Since $\operatorname{coker}(\eta) \cong \mathrm{O}_{X}$, the cone of $\eta$ induces a natural map $\psi: \mathfrak{O}_{X} \rightarrow \mathscr{O}_{X}[1]$. For all positive integers $n, \psi^{\otimes n}=\psi$. Clearly, $\psi$ vanishes at the points of $|X|$ (because $x^{*} \eta$ is split). If $\psi=\psi^{\otimes n}=0$ for some $n$, it is easily determined that this implies that $O_{X} \in \mathrm{D}_{\mathrm{qc}}(X)^{c}$, which is false.

Proof of Theorem 2.3. Let $\mathbf{E}$ be the category of representable, quasifinite, flat and separated morphisms of finite presentation over $X$. Let $\mathbf{D} \subseteq \mathbf{E}$ be the full subcategory whose objects are those $(U \stackrel{p}{\rightarrow} X)$ such that there exists an integer $n>0$ with $p^{*}\left(K \otimes_{\hat{O}_{X}}^{L}\left(\psi^{\otimes n}\right)\right)=0$. It suffices to prove that $\mathbf{D}=\mathbf{E}$. By the induction principle (Theorem B.1), it is sufficient to verify the following three conditions:

(I1) If ( $U \rightarrow W) \in \mathbf{E}$ is an open immersion and $W \in \mathbf{D}$, then $U \in \mathbf{D}$.

(I2) If ( $V \rightarrow W) \in \mathbf{E}$ is finite and surjective, where $V$ is an affine scheme, then $W \in \mathbf{D}$.

(I3) If $(U \stackrel{j}{\rightarrow} W),\left(W^{\prime} \stackrel{f}{\rightarrow} W\right) \in \mathbf{E}$, where $j$ is an open immersion and $f$ is étale and an isomorphism over $W \backslash U$, then $W \in \mathbf{D}$ whenever $U, W^{\prime} \in \mathbf{D}$.

Now condition (I1) is trivial and condition (I3) is Lemma 2.5. For condition (I2), by Lemma 2.6, it remains to prove that every affine scheme belongs to $\mathbf{D}$. By Lemma 2.2 and [Thomason 1997, Lemma 3.14] (or [Neeman 1992a, Lemma 1.2]), the result follows.

Lemma 2.5. Consider a 2-cartesian diagram of algebraic stacks

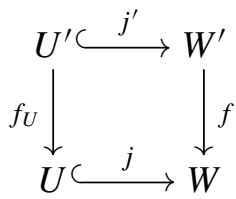

where $W$ is quasicompact and quasiseparated, $j$ is a quasicompact open immersion and $f$ is representable, étale, finitely presented and an isomorphism over $W \backslash U$. Let $\psi: E \rightarrow F$ be a morphism in $\mathrm{D}_{\mathrm{qc}}(W)$ and let $K \in \mathrm{D}_{\mathrm{qc}}(W)$. For each integer $n>0$, let $\phi_{n}=K \otimes_{\mathscr{O}_{W}}^{\llcorner}\left(\psi^{\otimes n}\right)$. If $f^{*} \phi_{n}=0$ and $j^{*} \phi_{n}=0$, then $\phi_{2 n}=0$.

Proof. To simplify notation, we let $E_{n}=K \otimes_{\mathscr{O}_{W}}^{\llcorner} E^{\otimes n}$ and $F_{n}=K \otimes_{\mathscr{O}_{W}}^{\mathrm{L}} F^{\otimes n}$. We will argue similarly to [Thomason 1997, Theorem 3.6], but using the MayerVietoris triangle for étale neighbourhoods of stacks developed in [Hall and Rydh 2014, Lemma 5.7(1)] instead of [Thomason 1997, Lemma 3.5]. Let $k=f \circ j^{\prime}$. By 
[Hall and Rydh 2014, Lemma 5.7(1)], there is a distinguished triangle in $\mathrm{D}_{\mathrm{qc}}(W)$ :

$$
F_{n} \longrightarrow \mathrm{R} j_{*} j^{*} F_{n} \oplus \mathrm{R} f_{*} f^{*} F_{n} \longrightarrow \mathrm{R} k_{*} k^{*} F_{n} \stackrel{d}{\longrightarrow} F_{n}[1] .
$$

By applying the homological functor $\operatorname{Hom}_{\mathscr{O}_{W}}\left(E_{n},-\right)$ to the distinguished triangle above, we find that there exists a morphism $t: E_{n} \rightarrow \mathrm{R} k_{*} k^{*} F_{n}[-1]$ such that $\delta(t)=\phi_{n}$, where $\delta$ is the boundary map induced by $d$. But there is a commutative diagram

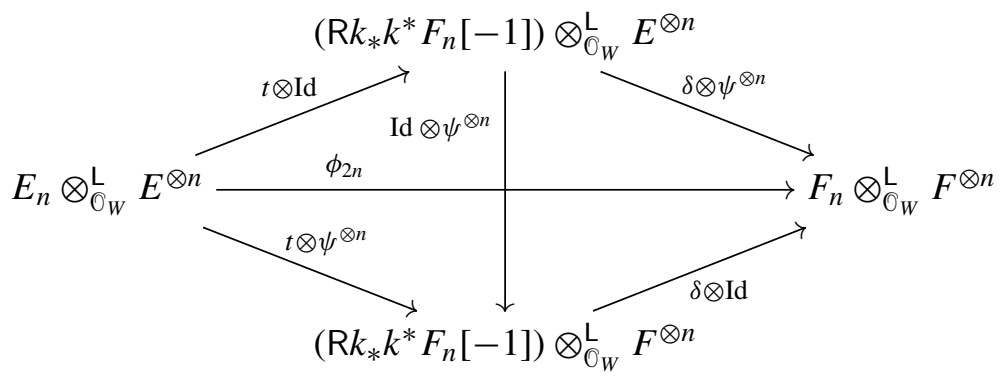

so it remains to prove that the vertical map above is zero. To see this, the projection formula [Hall and Rydh 2014, Corollary 4.12] implies that we have a commutative diagram

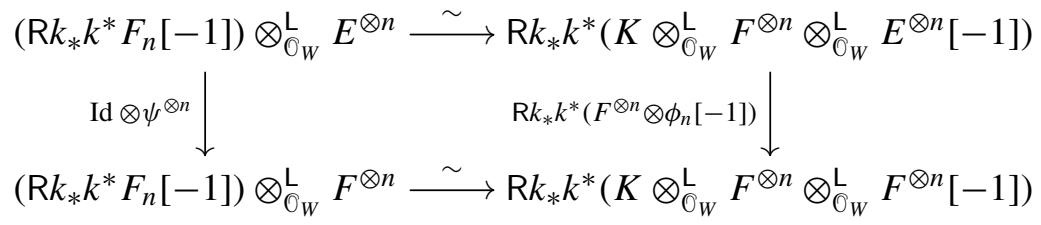

Since $k^{*} \phi_{n}=0$, the result follows.

The following lemma is similar to a special case of [Elagin 2011, Theorem 7.3 and Corollary 9.6]. Also, see [Krishna 2009, proof of Proposition 7.6; Dubey and Mallick 2012, Lemma 3.8].

Lemma 2.6. Let $W$ be an algebraic stack and let $v: V \rightarrow W$ be a finite and faithfully flat morphism of finite presentation, where $V$ is an affine scheme. Let $\psi: E \rightarrow F$ be a morphism in $\mathrm{D}_{\mathrm{qc}}(W)$, where $E \in \mathrm{D}_{\mathrm{qc}}(W)^{c}$. Let $K \in \operatorname{Perf}(W)$. If $v^{*}\left(K \otimes_{\hat{O}_{W}}^{\mathrm{L}} \psi\right)=0$ in $\mathrm{D}_{\mathrm{qc}}(V)$, then $K \otimes_{\hat{O}_{W}}^{\mathrm{L}} \psi=0$ in $\mathrm{D}_{\mathrm{qc}}(W)$.

Proof. By [Hall and Rydh 2014, Corollary 4.15], $\mathrm{R}\left(v_{\mathrm{qc}}\right)_{*}$ admits a right adjoint $v^{\times}$ and there is a functorial isomorphism $v^{\times}\left(\mathcal{O}_{W}\right) \otimes_{\mathscr{O}_{V}}^{\mathrm{L}} \mathrm{L} v_{\mathrm{qc}}^{*}(M) \simeq v^{\times}(M)$ for every $M \in \mathrm{D}_{\mathrm{qc}}(W)$. In particular, if $v^{*}\left(K \otimes_{\mathfrak{O}_{W}}^{\mathrm{L}} \psi\right)=0$ in $\mathrm{D}_{\mathrm{qc}}(V)$, then $v^{\times}\left(K \otimes_{\mathfrak{O}_{W}}^{\mathrm{L}} \psi\right)=0$ in $\mathrm{D}_{\mathrm{qc}}(V)$. By adjunction, it follows that the induced composition

$$
\mathrm{R}\left(v_{\mathrm{qc}}\right)_{*} v^{\times}\left(K \otimes_{\hat{O}_{W}}^{\mathrm{L}} E\right) \rightarrow K \otimes_{\hat{O}_{W}}^{\mathrm{L}} E \rightarrow K \otimes_{\hat{O}_{W}}^{\mathrm{L}} F
$$


vanishes in $\mathrm{D}_{\mathrm{qc}}(W)$. Thus it suffices to prove that

$$
\mathrm{R}\left(v_{\mathrm{qc}}\right)_{*} v^{\times}\left(K \otimes_{\hat{O}_{W}}^{\mathrm{L}} E\right) \rightarrow K \otimes_{\hat{\bigcirc}_{W}}^{\mathrm{L}} E
$$

admits a section. Since $E \in \mathrm{D}_{\mathrm{qc}}(W)^{c}$ and $K \in \operatorname{Perf}(W)$, it follows that $K \otimes_{\mathscr{O}_{W}}^{\mathrm{L}} E$ lies in $\mathrm{D}_{\mathrm{qc}}(W)^{c}$. Hence, we need only prove that if $M \in \mathrm{D}_{\mathrm{qc}}(W)^{c}$, the trace morphism $\operatorname{Tr}_{M}: \mathrm{R}\left(v_{\mathrm{qc}}\right)_{*} v^{\times}(M) \rightarrow M$ admits a section. By Lemma A.1, $M$ is quasi-isomorphic to a direct summand of $\mathrm{R}\left(v_{\mathrm{qc}}\right)_{*} P$, where $P \in \operatorname{Perf}(V)$. Thus we are reduced to proving that $\operatorname{Tr}_{\mathrm{R}\left(v_{\mathrm{qc}}\right)_{*} P}$ admits a section. This is trivial and the result follows.

\section{The classification of thick $\otimes$-ideals}

If $\mathscr{T}$ is a $\otimes$-triangulated category and $S \subseteq \mathscr{T}$ is a subset, then define $\langle S\rangle_{\otimes} \subseteq \mathscr{T}$ to be the smallest thick $\otimes$-ideal of $\mathcal{T}$ containing $S$.

To prove Theorem 1.1, we require this analogue of [Thomason 1997, Lemma 3.14]:

Lemma 3.1. Let $X$ be a quasicompact algebraic stack with quasifinite and separated diagonal. If $P, Q \in \mathrm{D}_{\mathrm{qc}}(X)^{c}$ and $\operatorname{supph}(P) \subseteq \operatorname{supph}(Q)$, then $\langle P\rangle_{\otimes} \subseteq\langle Q\rangle_{\otimes}$.

Proof. Argue exactly as in [Thomason 1997, Lemma 3.14] (cf. [Neeman 1992a, Lemma 1.2]), but using Theorem 2.3 instead of Thomason's Theorem 3.8.

The following example shows Lemma 3.1 cannot be extended to $P, Q \in \operatorname{Perf}(X)$ when $X$ is nontame. It also shows that Thomason's classification (Theorem 1.1) does not hold for $\operatorname{Perf}(X)$ in this case too.

Example 3.2. Let $x: \operatorname{Spec} \mathbb{F}_{2} \rightarrow X$ be as in Example 2.4. Let $P=\mathrm{O}_{X}$ and let $Q=x_{*} \mathrm{O}_{\operatorname{Spec}} \mathbb{F}_{2}$. Then $P, Q \in \operatorname{Perf}(X)$ and $\operatorname{supph}(P)=\operatorname{supph}(Q)$. Note that $Q \in \mathrm{D}_{\mathrm{qc}}(X)^{c}$ and $P \notin \mathrm{D}_{\mathrm{qc}}(X)^{c}$. Since $\mathrm{D}_{\mathrm{qc}}(X)^{c}$ is a thick $\otimes$-ideal of $\operatorname{Perf}(X)$, it follows that $\langle Q\rangle_{\otimes} \subseteq \mathrm{D}_{\mathrm{qc}}(X)^{c}$. But if $\langle P\rangle_{\otimes}=\langle Q\rangle_{\otimes}$, then $P \in \mathrm{D}_{\mathrm{qc}}(X)^{c}$. But $P \notin \mathrm{D}_{\mathrm{qc}}(X)^{c}$; thus we have a contradiction.

Following Thomason [1997, Theorem 3.15] (or Neeman [1992a, Theorem 1.5]), given Lemma 3.1, we can prove Theorem 1.1.

Proof of Theorem 1.1. If $Y \subseteq|X|$ is a Thomason subset, then define

$$
\mathscr{I}_{Y}=\left\{P \in \mathrm{D}_{\mathrm{qc}}(X)^{c}: \operatorname{supph}(P) \subseteq Y\right\} .
$$

Clearly, $\mathscr{I}_{Y}$ is a thick $\otimes$-ideal of $\mathrm{D}_{\mathrm{qc}}(X)^{c}$. If $\mathscr{T}$ is a thick $\otimes$-ideal of $\mathrm{D}_{\mathrm{qc}}(X)^{c}$, then define

$$
\varphi(\mathscr{T})=\bigcup_{Q \in \mathscr{T}} \operatorname{supph}(Q) .
$$

By [Hall and Rydh 2014, Lemma 4.8(3)], $\varphi(\mathscr{T})$ is a Thomason subset of $|X|$. It suffices to prove that $\Phi_{\varphi(\mathscr{T})}=\mathscr{T}$ and $\varphi\left(\mathscr{I}_{Y}\right)=Y$. 
Obviously, $\mathscr{T} \subseteq \mathscr{I}_{\varphi(\mathcal{T})}$. For the reverse inclusion, if $P \in \mathscr{I}_{\varphi(\mathcal{T})}$, then

$$
\operatorname{supph}(P) \subseteq \bigcup_{Q \in \mathscr{T}} \operatorname{supph}(Q) .
$$

Since $\operatorname{supph}(P)$ and $\operatorname{supph}(Q)$ are constructible for every $Q \in \mathcal{T}$, it follows that there is a finite subset $J \subseteq \mathcal{T}$ such that

$$
\operatorname{supph}(P) \subseteq \bigcup_{Q \in J} \operatorname{supph}(Q)=\operatorname{supph}\left(\oplus_{Q \in J} Q\right) .
$$

By Lemma 3.1, $\langle P\rangle_{\otimes} \subseteq\left\langle\oplus_{Q \in J} Q\right\rangle_{\otimes} \subseteq \mathscr{T}$. Thus $P \in \mathscr{T}$ and $\mathscr{I}_{\varphi(\mathscr{T})}=\mathscr{T}$.

Obviously, $Y \supseteq \varphi\left(\mathscr{I}_{Y}\right)$. Since $Y$ is Thomason, it is expressible as a union $\cup_{\alpha} Y_{\alpha}$ such that $|X| \backslash Y_{\alpha}$ is quasicompact and open. By [Hall and Rydh 2014, Theorem A], for every $\alpha$ there is a compact complex $Q_{\alpha}$ with support $Y_{\alpha}$. It follows that if $y \in Y$, then $y \in \operatorname{supph}\left(Q_{\alpha}\right) \subseteq Y$ for some $\alpha$. In other words, $y \in \varphi\left(\mathscr{I}_{Y}\right)$, so $Y=\varphi\left(\mathscr{I}_{Y}\right)$.

\section{The Balmer spectrum of a tame stack}

We will prove Theorem 1.2 using [Buan et al. 2007, Proposition 6.1].

Proof of Theorem 1.2. Let $s:(|X|$, supph $) \rightarrow\left(\left|\operatorname{Sp}_{\text {Bal }}(\operatorname{Perf}(X))\right|, \sigma_{X}\right)$ be the uniquely induced morphism of support data, where $\sigma_{X}$ denotes the universal support datum. Since $X$ is tame, it has finite cohomological dimension [Hall and Rydh 2015, Theorem 2.1(2)]; hence, $\mathrm{D}_{\mathrm{qc}}(X)^{c}=\operatorname{Perf}(X)$ [Hall and Rydh 2014, Remark 4.6]. By Theorem 1.1, $(|X|$, supph) is classifying and by [Laumon and Moret-Bailly 2000, Corollaries 5.6.1 and 5.7.2] we know that $|X|$ is spectral. By [Buan et al. 2007, Proposition 6.1], $s$ is a homeomorphism. By definition, $O_{\mathrm{Sp}_{\mathrm{Bal}}(\operatorname{Perf}(X))}$ is the sheafification of the presheaf

$$
(j: U \subseteq X) \mapsto \operatorname{End}_{\operatorname{Perf}(X) / \operatorname{ker}\left(j^{*}\right) \cap \operatorname{Perf}(X)}\left(j^{*} \mathcal{O}_{X}\right) .
$$

Since $|X|$ has a basis consisting of quasicompact open subsets, it is sufficient to identify $\operatorname{End}_{\operatorname{Perf}(X) / \operatorname{ker}\left(j^{*}\right) \cap \operatorname{Perf}(X)}\left(j^{*} O_{X}\right)$ when $j$ is a quasicompact open immersion. By [Hall and Rydh 2014, Lemma 6.7(2)], $\operatorname{ker}\left(j^{*}\right)$ is the localising envelope of a set of objects with compact image in $\mathrm{D}_{\mathrm{qc}}(X)$. By Thomason's localisation theorem (e.g., [Hall and Rydh 2014, Theorem 3.10] or [Neeman 1992b, Theorem 2.1]), $\operatorname{Perf}(U)$ is the thick closure of $\operatorname{Perf}(X) / \operatorname{ker}\left(j^{*}\right) \cap \operatorname{Perf}(X)$. Since there are natural isomorphisms

$$
\operatorname{End}_{\operatorname{Perf}(X) / \operatorname{ker}\left(j^{*}\right) \cap \operatorname{Perf}(X)}\left(j^{*} \mathscr{O}_{X}\right) \cong \operatorname{End}_{\operatorname{Perf}(U)}\left(\mathscr{O}_{U}\right) \cong \operatorname{End}_{\mathscr{O}_{U}}\left(\mathscr{O}_{U}\right)=\Gamma\left(U, \mathscr{O}_{X}\right),
$$

the result follows.

Proof of Theorem 1.3. Since $X$ has finite inertia, it has separated diagonal. By [Rydh 2013, Theorem 6.12], $\pi$ is a separated universal homeomorphism, so $X_{\mathrm{cs}}$ is a quasicompact and quasiseparated algebraic space. By [Rydh 2013, Theorem 6.12], 
the natural map $\left(|X|, \mathrm{O}_{X_{\mathrm{Zar}}}\right) \rightarrow\left(\left|X_{\mathrm{cs}}\right|, \mathrm{O}_{\left(X_{\mathrm{cs}}\right)_{\mathrm{Zar}}}\right)$ is an isomorphism of locally ringed spaces. By Theorem 1.2, the result follows.

\section{Appendix A: Tame stacks and coarse spaces}

We establish here some basic results about $\mathrm{R}\left(\pi_{\mathrm{qc}}\right)_{*}$, where $\pi: X \rightarrow X_{\mathrm{cs}}$ is the coarse space of a quasiseparated algebraic stack $X$ with finite inertia. Our first result, however, is a useful lemma that characterises the compact objects on a certain class of algebraic stacks, which includes $B G$ for all finite groups $G$. This is likely known, though we are unaware of a reference for this result in the generality required.

Lemma A.1. Let $W$ be an algebraic stack and let $v: V \rightarrow W$ be a finite and faithfully flat morphism of finite presentation, where $V$ is an affine scheme. If $M \in \mathrm{D}_{\mathrm{qc}}(W)^{c}$, then $M$ is quasi-isomorphic to a direct summand of $\mathrm{R}\left(v_{\mathrm{qc}}\right)_{*} P$ for some $P \in \operatorname{Perf}(V)$.

Proof. If $P \in \operatorname{Perf}(V)$, then $\mathrm{R}\left(v_{\mathrm{qc}}\right)_{*} P \in \mathrm{D}_{\mathrm{qc}}(W)^{c}$ [Hall and Rydh 2014, Corollary 4.15 and Example 3.8]. Thus, let $\mathscr{T} \subseteq \mathrm{D}_{\mathrm{qc}}(W)^{c}$ be the subcategory with objects those $N \in \mathrm{D}_{\mathrm{qc}}(W)^{c}$ that are quasi-isomorphic to direct summands of $\mathrm{R}\left(v_{\mathrm{qc}}\right)_{*} P$ for some $P \in \operatorname{Perf}(V)$. Clearly, $\mathscr{T}$ is closed under shifts and direct summands. We now prove that $\mathscr{T}$ is triangulated. Thus let $f: N^{\prime} \rightarrow N$ be a morphism in $\mathscr{T}$ and complete it to a distinguished triangle

$$
N^{\prime} \stackrel{f}{\longrightarrow} N \stackrel{c}{\longrightarrow} N^{\prime \prime} \stackrel{\partial}{\longrightarrow} N^{\prime}[1] .
$$

We now prove that $N^{\prime \prime} \in \mathscr{T}$. By assumption, there are $P, P^{\prime} \in \operatorname{Perf}(V)$ and $C$, $C^{\prime} \in \mathrm{D}_{\mathrm{qc}}(W)^{c}$ and quasi-isomorphisms $N \oplus C \simeq \mathrm{R}\left(v_{\mathrm{qc}}\right)_{*} P, N^{\prime} \oplus C^{\prime} \simeq \mathrm{R}\left(v_{\mathrm{qc}}\right)_{*} P^{\prime}$. It follows that there is a distinguished triangle

$$
N^{\prime} \oplus C^{\prime} \stackrel{f \oplus 0}{\longrightarrow} N \oplus C \stackrel{c \oplus \mathrm{id}_{C} \oplus 0}{\longrightarrow} N^{\prime \prime} \oplus C \oplus C^{\prime}[1] \stackrel{\partial \oplus p_{C^{\prime}[1]}}{\longrightarrow} N^{\prime} \oplus C^{\prime}[1],
$$

where $p_{C^{\prime}[1]}: C \oplus C^{\prime}[1] \rightarrow C^{\prime}[1]$ is the natural projection. In particular, we are reduced to the situation where $N^{\prime}=\mathrm{R}\left(v_{\mathrm{qc}}\right)_{*} P^{\prime}$ and $N=\mathrm{R}\left(v_{\mathrm{qc}}\right)_{*} P$. In this case, the morphism $f: N^{\prime} \rightarrow N$ by duality induces a morphism $\tilde{f}: P^{\prime} \rightarrow v^{\times} \mathrm{R}\left(v_{\mathrm{qc}}\right)_{*} P$. It follows that the composition $\mathrm{R}\left(v_{\mathrm{qc}}\right)_{*} P^{\prime} \stackrel{f}{\rightarrow} \mathrm{R}\left(v_{\mathrm{qc}}\right)_{*} P \rightarrow \mathrm{R}\left(v_{\mathrm{qc}}\right)_{*} v^{\times} \mathrm{R}\left(v_{\mathrm{qc}}\right)_{*} P$ is the map $\mathrm{R}\left(v_{\mathrm{qc}}\right)_{*} \tilde{f}$. Now form a distinguished triangle

$$
P^{\prime} \stackrel{\tilde{f}}{\longrightarrow} v^{\times} \mathrm{R}\left(v_{\mathrm{qc}}\right)_{*} P \stackrel{k}{\longrightarrow} K \stackrel{\delta}{\longrightarrow} P^{\prime}[1] .
$$

Since the morphism $\mathrm{R}\left(v_{\mathrm{qc}}\right)_{*} P \rightarrow \mathrm{R}\left(v_{\mathrm{qc}}\right)_{*} v^{\times} \mathrm{R}\left(v_{\mathrm{qc}}\right)_{*} P$ admits a retraction, there exist a $Q \in \mathrm{D}_{\mathrm{qc}}(W)^{c}$ and a quasi-isomorphism $\mathrm{R}\left(v_{\mathrm{qc}}\right)_{*} v^{\times} \mathrm{R}\left(v_{\mathrm{qc}}\right)_{*} P \simeq \mathrm{R}\left(v_{\mathrm{qc}}\right)_{*} P \oplus Q$. 
There is an induced morphism of distinguished triangles

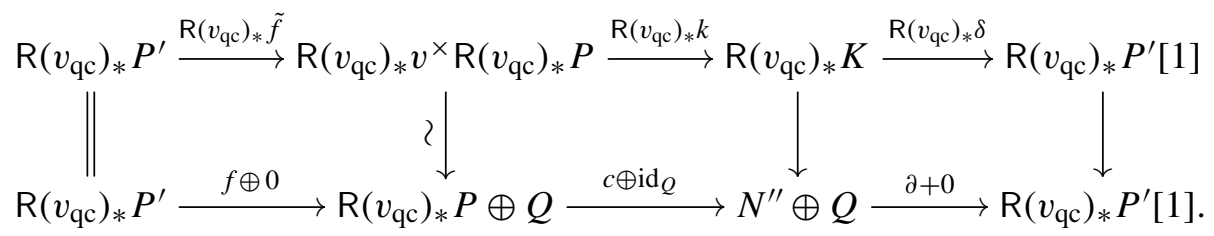

It follows that $\mathrm{R}\left(v_{\mathrm{qc}}\right)_{*} K \simeq N^{\prime \prime} \oplus Q$ and so $N^{\prime \prime} \in \mathscr{T}$. By [Hall and Rydh 2014, Example 6.5 and Proposition 6.6], $\mathrm{D}_{\mathrm{qc}}(W)$ is compactly generated by $v_{*} \mathcal{O}_{V}$. But Thomason's Theorem [Neeman 1992b, Theorem 2.1] implies that $\mathrm{D}_{\mathrm{qc}}(W)^{c}$ is the smallest thick subcategory containing $v_{*} \mathrm{O}_{V}$. The result follows.

Let $F: \mathscr{S} \rightarrow \mathscr{T}$ be a triangulated functor between triangulated categories. Assume that $\mathscr{Y}$ and $\mathscr{T}$ admit $t$-structures. We say that $F$ is left (resp. right) $t$-exact if $F\left(\mathscr{S}^{\geq 0}\right) \subseteq \mathscr{T}^{\geq 0}$ (resp. $\left.F\left(\mathscr{S}^{\leq 0}\right) \subseteq \mathscr{T}^{\leq 0}\right)$ ). We say that $F$ is $t$-exact if it is both left and right $t$-exact. The following result was suggested to us by David Rydh.

Theorem A.2. If $X$ be a quasiseparated algebraic stack with finite inertia and coarse space $\pi: X \rightarrow X_{\mathrm{cs}}$, then the restriction of $\mathrm{R}\left(\pi_{\mathrm{qc}}\right)_{*}$ to $\mathrm{D}_{\mathrm{qc}}(X)^{c}$ is $t$-exact.

Proof. By [Hall and Rydh 2014, Lemma 1.2(4)], this may be checked étale-locally on $X_{\mathrm{cs}}$. Thus, we may assume that $X_{\mathrm{cs}}$ is an affine scheme. Since $\pi$ is a universal homeomorphism, it follows that $X$ is quasicompact. Also, since $X$ has finite inertia, it has quasifinite and separated diagonal. By Theorem B.5, there exist morphisms of algebraic stacks $V \stackrel{v}{\rightarrow} W \stackrel{p}{\rightarrow} X$ such that $V$ is an affine scheme, $v$ is finite, faithfully flat and finitely represented and $p$ is a representable, separated and finitely presented Nisnevich covering. By [Rydh 2013, Proposition 6.5], we may further assume that $p$ is fixed-point reflecting. We now apply [Rydh 2013, Theorem 6.10] to conclude that the diagram

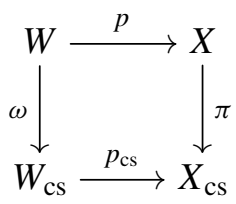

is cartesian and $p_{\mathrm{cs}}$ is representable, separated, étale and of finite presentation. Thus, it suffices to prove the result on $W$.

Clearly $\mathrm{R}\left(\pi_{\mathrm{qc}}\right)_{*}$ is left $t$-exact, so it remains to address the right $t$-exactness. Take $M \in \mathrm{D}_{\mathrm{qc}}(W)^{c} \cap \mathrm{D}_{\mathrm{qc}}^{\leq 0}(W)$. By Lemma A.1, we may assume that there exists a map $i: M \rightarrow \mathrm{R}\left(v_{\mathrm{qc}}\right)_{*} P$, where $P \in \operatorname{Perf}(V)$, that admits a retraction $r$. It follows that the composition $M \stackrel{i}{\rightarrow} \mathrm{R}\left(v_{\mathrm{qc}}\right)_{*} P \rightarrow \tau^{>0} \mathrm{R}\left(v_{\mathrm{qc}}\right)_{*} P$ is the zero map. Therefore the induced map $\mathrm{R}\left(\omega_{\mathrm{qc}}\right)_{*} M \rightarrow \mathrm{R}\left(\omega_{\mathrm{qc}}\right)_{*} \tau^{>0} \mathrm{R}\left(v_{\mathrm{qc}}\right)_{*} P$ is the zero map. But $v$ and 
$\omega \circ v$ are affine, so there is a natural quasi-isomorphism $\tau^{>0} \mathrm{R}\left(\omega_{\mathrm{qc}}\right)_{*} \mathrm{R}\left(v_{\mathrm{qc}}\right)_{*} P \simeq$ $\mathrm{R}\left(\omega_{\mathrm{qc}}\right)_{*} \tau^{>0} \mathrm{R}\left(v_{\mathrm{qc}}\right)_{*} P$. The resulting map

$$
\tau^{>0} \mathrm{R}\left(\omega_{\mathrm{qc}}\right)_{*} M \rightarrow \tau^{>0} \mathrm{R}\left(\omega_{\mathrm{qc}}\right)_{*} \mathrm{R}\left(v_{\mathrm{qc}}\right)_{*} P
$$

is 0 and also coincides with $\tau^{>0} \mathrm{R}\left(\omega_{\mathrm{qc}}\right)_{*}(i)$, which admits a retraction $\tau^{>0} \mathrm{R}\left(\omega_{\mathrm{qc}}\right)_{*}(r)$. In particular, $\tau^{>0} \mathrm{R}\left(\omega_{\mathrm{qc}}\right)_{*} M \simeq 0$ and the result follows.

Abramovich et al. [2008] work with a more restrictive definition of tame, rendering the following corollary a tautology. Indeed, they assume that $X$ has finite inertia and is locally of finite presentation over a base scheme $S$ and that $\pi: X \rightarrow X_{\mathrm{cs}}$ is such that $\pi_{*}$ is exact on quasicoherent sheaves. In our case, we make none of these assumptions, rendering it nontrivial.

Corollary A.3. Let $X$ be a quasiseparated algebraic stack with finite inertia and coarse space $\pi: X \rightarrow X_{\mathrm{cs}}$. The following are equivalent:

(1) $X$ is tame;

(2) $\pi_{*}: \mathrm{Q} \operatorname{Coh}(X) \rightarrow \mathrm{Q} \operatorname{Coh}\left(X_{\mathrm{cs}}\right)$ is exact;

(3) $\mathrm{R} \pi_{*}: \mathrm{D}_{\mathrm{qc}}^{+}(X) \rightarrow \mathrm{D}_{\mathrm{qc}}^{+}\left(X_{\mathrm{cs}}\right)$ is $t$-exact;

(4) $\mathrm{R}\left(\pi_{\mathrm{qc}}\right)_{*}: \mathrm{D}_{\mathrm{qc}}(X) \rightarrow \mathrm{D}_{\mathrm{qc}}\left(X_{\mathrm{cs}}\right)$ is $t$-exact.

Proof. We begin with some preliminary reductions. The morphism $\pi$ is a separated universal homeomorphism [Rydh 2013, Theorem 6.12], so $X_{\mathrm{cs}}$ is a quasiseparated algebraic space and $\pi$ is quasicompact and quasiseparated. Thus by Lemma 1.2(2) of [Hall and Rydh 2014] we get the implication (3) $\Rightarrow(4)$, and by Theorem 2.6(2) of the same reference we have that (4) $\Rightarrow(3)$. Clearly, item (1) may be verified after passing to an affine étale presentation of $X_{\mathrm{cs}}$, and similarly for items (2) and (3) [Hall and Rydh 2014, Lemma 1.2(4) and Lemma 2.2(6)]. We may consequently assume that $X_{\mathrm{cs}}$ is an affine scheme. Since $\pi$ has finite diagonal, it has affine diagonal, so we have (2) $\Leftrightarrow(3)$ [Hall et al. 2014, Proposition 2.1]. By [Hall and Rydh 2015, Theorem C, (1) $\Rightarrow(3)$ ], we now obtain that $(2) \Rightarrow(1)$. It remains to address $(1) \Rightarrow(2)$.

Arguing exactly as in the proof of Theorem A.2, we may further assume that $X$ admits a finite, faithfully flat and finitely presented cover $v: V \rightarrow X$, where $V$ is an affine scheme. Since $X$ is tame, $\mathscr{O}_{X} \in \mathrm{D}_{\mathrm{qc}}(X)^{c}$. By Theorem A.2, it follows that the induced morphism $\mathbb{O}_{X} \rightarrow v_{*} \hat{O}_{V}$ admits a retraction. If $M \in \mathrm{Q} \operatorname{Coh}(X)$, then it follows immediately that the natural map $M \rightarrow v_{*} v^{*} M$ admits a retraction. Thus, if $f: M \rightarrow N$ is a surjection in QCoh(X), then $f$ is a retraction of the surjection $v_{*} v^{*} f$. Since $\pi \circ v$ is affine, $\pi_{*} v_{*} v^{*} f$ is surjective. In particular, $\pi_{*} f$ is a retraction of a surjection, thus is surjective. The result follows. 


\section{Appendix B: The induction principle}

The induction principle [Stacks 2015, Tag 08GL] for algebraic spaces is closely related to the étale dévissage results of [Rydh 2011a]. When working with derived categories, where locality results are often quite subtle, it is often advantageous to have the strongest possible criteria at your disposal. In this appendix, we will prove the following induction principle for stacks with quasifinite and separated diagonal.

Before stating this result, we require some notation. Fix an algebraic stack $S$. If $P_{1}, \ldots, P_{r}$ is a list of properties of morphisms of algebraic stacks over $S$, let Stack $_{P_{1}, \ldots, P_{r} / S}$ denote the full 2-subcategory of the category of algebraic stacks over $S$ whose objects are those $(x: X \rightarrow S)$ such that $x$ has properties $P_{1}, \ldots, P_{r}$. The following abbreviations will be used: ét (étale), qff (quasifinite flat), sep (separated), fp (finitely presented) and rep (representable).

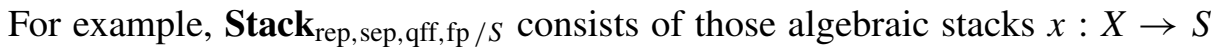
such that $x$ is representable, separated, quasifinite flat, and finitely presented. In a similar way, Stack $_{\text {rep,sep,ét,fp } / S}$ consists of those algebraic stacks over $S, x: X \rightarrow S$, such that $x$ is representable, separated, étale, and finitely presented. Note that

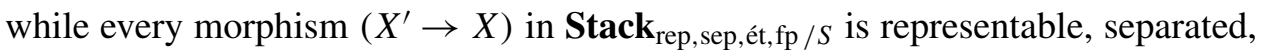
étale, and finitely presented; in $\mathbf{S t a c k}_{\mathrm{rep}, \mathrm{sep}, \mathrm{qff}, \mathrm{fp} / S}$ they can only be assumed to be representable, separated, quasifinite, and finitely presented (i.e., there are nonflat morphisms between objects).

Theorem B.1 (induction principle). Let $S$ be a quasicompact algebraic stack with quasicompact and separated diagonal. If $S$ has quasifinite diagonal, let

$$
\mathbf{E}=\text { Stack }_{\text {rep }, \text { sep }, \mathrm{qff}, \mathrm{fp} / S}
$$

or if $S$ is Deligne-Mumford, let

$$
\mathbf{E}=\mathbf{S t a c k}_{\text {rep }, \text { sep }, \text { ét,fp } / S} .
$$

Let $\mathbf{D} \subseteq \mathbf{E}$ be a full subcategory satisfying the following properties:

(I1) if $\left(X^{\prime} \rightarrow X\right) \in \mathbf{E}$ is an open immersion and $X \in \mathbf{D}$, then $X^{\prime} \in \mathbf{D}$;

(I2) if $\left(X^{\prime} \rightarrow X\right) \in \mathbf{E}$ is finite, flat, and surjective, where $X^{\prime}$ is an affine scheme, then $X \in \mathbf{D}$;

(I3) if $(U \stackrel{j}{\rightarrow} X),\left(X^{\prime} \stackrel{f}{\rightarrow} X\right) \in \mathbf{E}$, where $j$ is an open immersion and $f$ is étale and an isomorphism over $X \backslash U$, then $X \in \mathbf{D}$ whenever $U, X^{\prime} \in \mathbf{D}$.

Then $\mathbf{D}=\mathbf{E}$. In particular, $S \in \mathbf{D}$.

Proof. Combine Lemma B.3 with Theorem B.5. 
We wish to point out that Theorem B.1 relies on the existence of coarse spaces for stacks with finite inertia (i.e., the Keel-Mori theorem [Keel and Mori 1997; Rydh 2013]).

Nisnevich coverings. It will be useful to consider some variants and refinements of [Krishna and Østvær 2012, Sections 7-8].

If $p: W \rightarrow X$ is a representable morphism of algebraic stacks, then a splitting sequence for $p$ is a sequence of quasicompact open immersions

$$
\varnothing=X_{0} \subseteq X_{1} \subseteq \cdots \subseteq X_{r}=X
$$

such that $p$ restricted to $X_{i} \backslash X_{i-1}$, when given the induced reduced structure, admits a section for each $i=1, \ldots, r$. In this situation, we say that $p$ has a splitting sequence of length $r$. An étale and representable morphism of algebraic stacks $p: W \rightarrow X$ is a Nisnevich covering if it admits a splitting sequence.

Example B.2. Let $X$ be a quasicompact and quasiseparated scheme. Then there exists an affine scheme $W$ and a Nisnevich covering $p: W \rightarrow X$. Indeed, taking $W=\amalg_{i=1}^{n} U_{i}$, where the $\left\{U_{i}\right\}$ form a finite affine open covering of $X$ gives the claim.

The following lemma is proved by a straightforward induction on the length of the splitting sequence.

Lemma B.3 (Nisnevich dévissage). Let $S$ be a quasicompact and quasiseparated

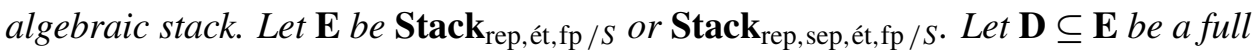
2-subcategory with the following properties:

(N1) if $\left(X^{\prime} \rightarrow X\right) \in \mathbf{E}$ is an open immersion and $X \in \mathbf{D}$, then $X^{\prime} \in \mathbf{D}$;

(N2) if $(U \stackrel{j}{\rightarrow} X),\left(X^{\prime} \stackrel{f}{\rightarrow} X\right) \in \mathbf{E}$, where $j$ is an open immersion and $f$ is an isomorphism over $X \backslash U$, then $X \in \mathbf{D}$ whenever $U, X^{\prime} \in \mathbf{D}$.

If $p: W \rightarrow X$ is a Nisnevich covering in $\mathbf{E}$ and $W \in \mathbf{D}$, then $X \in \mathbf{D}$.

The following lemma will also be useful.

Lemma B.4. Let $p: W \rightarrow X$ be a Nisnevich covering of algebraic stacks.

(1) If $f: X^{\prime} \rightarrow X$ is a morphism of algebraic stacks, then the pull back $p^{\prime}: W^{\prime} \rightarrow X^{\prime}$ of $p$ along $f$ is a Nisnevich covering.

(2) Let $w: W^{\prime} \rightarrow W$ be a Nisnevich covering of finite presentation. If $p$ is of finite presentation and $X$ is quasicompact and quasiseparated, then $p \circ w: W^{\prime} \rightarrow X$ is a Nisnevich covering. 
Presentations. The following theorem refines [Rydh 2011a, Theorem 7.2] and will be crucial for the proof of Theorem B.1.

Theorem B.5. Let $X$ be a quasicompact algebraic stack with quasifinite and separated diagonal. Then there exist morphisms of algebraic stacks

$$
V \stackrel{v}{\rightarrow} W \stackrel{p}{\rightarrow} X
$$

such that

- $V$ is an affine scheme;

- $v$ is finite, flat, surjective and of finite presentation;

- $p$ is a separated Nisnevich covering of finite presentation.

In addition, if $S$ is a Deligne-Mumford stack, it can be arranged that $v$ is also étale.

Proof. The proof is similar to [Rydh 2013, Proposition 6.11; 2011a, Theorem 7.3].

By [Rydh 2011a, Theorem 7.1], there is an affine scheme $U$ and a representable, separated, quasifinite, flat, and surjective morphism $u: U \rightarrow X$ of finite presentation. Let $W=\underline{\text { Hilb }}_{U / X}^{\text {open }} \rightarrow X$ be the subfunctor of the relative Hilbert scheme parametrising open and closed immersions to $U$ over $X$. It follows that $p: W \rightarrow X$ is étale, representable and separated [Rydh 2011b, Corollary 6.2].

We now prove that $p$ is a Nisnevich covering. To see this, we note that there exists a sequence of quasicompact open immersions

$$
\varnothing=X_{0} \subseteq X_{1} \subseteq \cdots \subseteq X_{r}=X,
$$

such that the restriction of $u$ to $Z_{i}=\left(X_{i} \backslash X_{i-1}\right)_{\text {red }}$ for $i=1, \ldots, r$ is finite, flat and finitely presented. By definition of $p: W \rightarrow X$, it follows immediately that $p \mid z_{i}$ admits a section corresponding to $\left.u\right|_{Z_{i}}$ and so $p$ is a separated Nisnevich covering.

Let $v: V \rightarrow W$ be the universal family, which is finite, flat, surjective and of finite presentation. Also, $V \rightarrow U$ is representable, étale and separated [Rydh 2011b, Corollary 6.2]. Suitably shrinking $W$, we obtain a separated Nisnevich covering $p: W \rightarrow X$ of finite presentation fitting into a 2-commutative diagram

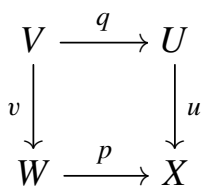

and $q$ is étale, separated and surjective. By Zariski's Main Theorem [Laumon and Moret-Bailly 2000, Theorem A.2], $q$ is quasi-affine. By [Rydh 2013, Theorem 5.3], $W$ has a coarse space $\pi: W \rightarrow W_{\mathrm{cs}}$ such that $W_{\mathrm{cs}}$ is a quasi-affine scheme and $\pi \circ v$ 
is affine. By Example B.2 and Lemma B.4, we may further reduce to the situation where $W_{\mathrm{cs}}$ is an affine scheme. Since $\pi \circ v$ is affine, the result follows.

\section{Acknowledgements}

I would like to thank Amnon Neeman, Ben Antieau, Ravi Vakil, and Olaf Schnürer for their encouraging remarks and suggestions. I would especially like to thank David Rydh for sharing some insights on tame stacks and their coarse moduli spaces. A special thanks is due to the referee for some very useful comments and corrections.

\section{References}

[Abramovich et al. 2008] D. Abramovich, M. Olsson, and A. Vistoli, "Tame stacks in positive characteristic", Ann. Inst. Fourier (Grenoble) 58:4 (2008), 1057-1091. MR 2009c:14002 Zbl 1222.14004

[Balmer 2005] P. Balmer, "The spectrum of prime ideals in tensor triangulated categories", J. Reine Angew. Math. 588 (2005), 149-168. MR 2007b:18012 Zbl 1080.18007

[Balmer 2013] P. Balmer, "Separable extensions in tensor-triangular geometry and generalized Quillen stratification”, preprint, 2013. To appear in Ann. Sci. École Norm. Sup. (4). arXiv 1309.1808

[Benson et al. 2011] D. J. Benson, S. B. Iyengar, and H. Krause, "Stratifying modular representations of finite groups", Ann. of Math. (2) 174:3 (2011), 1643-1684. MR 2846489 Zbl 1261.20057

[Buan et al. 2007] A. B. Buan, H. Krause, and Ø. Solberg, "Support varieties: an ideal approach", Homology, Homotopy Appl. 9:1 (2007), 45-74. MR 2008i:18007 Zbl 1118.18005

[Dubey and Mallick 2012] U. V. Dubey and V. M. Mallick, "Spectrum of some triangulated categories", J. Algebra 364 (2012), 90-118. MR 2927050 Zbl 1258.14020

[Elagin 2011] A. D. Elagin, “Когомологическая теория спуска для морфизма стеков и для эквивариантных производных категорий”, Mat. Sb. 202:4 (2011), 31-64. Translated as "Cohomological descent theory for a morphism of stacks and for equivariant derived categories" in Sbornik Math. 202:4 (2011), 495-526. MR 2012e:14040 Zbl 1234.18006

[Hall and Rydh 2014] J. Hall and D. Rydh, "Perfect complexes on algebraic stacks", preprint, 2014. arXiv $1405.1887 \mathrm{v} 2$

[Hall and Rydh 2015] J. Hall and D. Rydh, "Algebraic groups and compact generation of their derived categories of representations", Indiana Univ. Math. J. 64:6 (2015), 1903-1923.

[Hall et al. 2014] J. Hall, A. Neeman, and D. Rydh, "One positive and two negative results for derived categories of algebraic stacks", preprint, 2014. arXiv 1405.1888

[Hopkins 1987] M. J. Hopkins, "Global methods in homotopy theory", pp. 73-96 in Homotopy theory (Durham, 1985), edited by E. Rees and J. D. S. Jones, London Mathematical Society Lecture Note Series 117, Cambridge University Press, 1987. MR 89g:55022 Zbl 0657.55008

[Keel and Mori 1997] S. Keel and S. Mori, "Quotients by groupoids", Ann. of Math. (2) 145:1 (1997), 193-213. MR 97m:14014 Zbl 0881.14018

[Knutson 1971] D. Knutson, Algebraic spaces, Lecture Notes in Mathematics 203, Springer, Berlin, 1971. MR 46\#1791 Zbl 0221.14001

[Krishna 2009] A. Krishna, "Perfect complexes on Deligne-Mumford stacks and applications", J. K-Theory 4:3 (2009), 559-603. MR 2011d:19011 Zbl 1189.19003 
[Krishna and Østvær 2012] A. Krishna and P. A. Østvær, "Nisnevich descent for $K$-theory of DeligneMumford stacks”, J. K-Theory 9:2 (2012), 291-331. MR 2922391 Zbl 1284.19005

[Laumon and Moret-Bailly 2000] G. Laumon and L. Moret-Bailly, Champs algébriques, Ergebnisse der Mathematik und ihrer Grenzgebiete (3) 39, Springer, Berlin, 2000. MR 2001f:14006 Zbl 0945.14005

[Neeman 1992a] A. Neeman, "The chromatic tower for $D(R)$ ", Topology 31:3 (1992), 519-532. MR 93h:18018 Zbl 0793.18008

[Neeman 1992b] A. Neeman, "The connection between the $K$-theory localization theorem of Thomason, Trobaugh and Yao and the smashing subcategories of Bousfield and Ravenel", Ann. Sci. École Norm. Sup. (4) 25:5 (1992), 547-566. MR 93k:18015 Zbl 0868.19001

[Neeman 2015] A. Neeman, "Separable monoids in $\mathrm{D}_{\mathrm{qc}}(X)$ ", J. Reine Angew. Math. (online publication August 2015).

[Rydh 2011a] D. Rydh, "Étale dévissage, descent and pushouts of stacks", J. Algebra 331 (2011), 194-223. MR 2012d:14002 Zbl 1230.14005

[Rydh 2011b] D. Rydh, "Representability of Hilbert schemes and Hilbert stacks of points", Comm. Algebra 39:7 (2011), 2632-2646. MR 2012j:14007 Zbl 1235.14007

[Rydh 2013] D. Rydh, "Existence and properties of geometric quotients", J. Algebraic Geom. 22:4 (2013), 629-669. MR 3084720 Zbl 1278.14003

[Stacks 2015] A. J. de Jong, P. Belmans, et al., "The stacks project", 2015, Available at http:// math.columbia.edu/algebraic_geometry/stacks-git.

[Thomason 1997] R. W. Thomason, "The classification of triangulated subcategories", Compositio Math. 105:1 (1997), 1-27. MR 98b:18017 Zbl 0873.18003

Received 29 Dec 2014. Revised 6 Oct 2015. Accepted 4 Nov 2015.

JACK HALL: jack.hall@anu.edu.au

Mathematical Sciences Institute, The Australian National University, Acton ACT 2601, Australia 


\title{
ANNALS OF K-THEORY
}

\author{
msp.org/akt
}

EDITORIAL BOARD

\author{
Paul Balmer University of California, Los Angeles, USA \\ balmer@math.ucla.edu \\ Spencer Bloch University of Chicago, USA \\ bloch@math.uchicago.edu \\ Alain Connes Collège de France; Institut des Hautes Études Scientifiques; Ohio State University \\ alain@connes.org \\ Guillermo Cortiñas Universidad de Buenos Aires and CONICET, Argentina \\ gcorti@dm.uba.ar \\ Eric Friedlander University of Southern California, USA \\ ericmf@usc.edu \\ Max Karoubi Institut de Mathématiques de Jussieu - Paris Rive Gauche, France \\ max.karoubi@imj-prg.fr \\ Gennadi Kasparov Vanderbilt University, USA \\ gennadi.kasparov@vanderbilt.edu \\ Alexander Merkurjev \\ University of California, Los Angeles, USA \\ merkurev@math.ucla.edu \\ Amnon Neeman amnon.Australian National University \\ neeman@anu.edu.au \\ Jonathan Rosenberg (Managing Editor) \\ University of Maryland, USA \\ jmr@math.umd.edu \\ Marco Schlichting University of Warwick, UK \\ schlichting@warwick.ac.uk \\ Andrei Suslin Northwestern University, USA \\ suslin@math.northwestern.edu \\ Vladimir Voevodsky Institute for Advanced Studies, USA \\ vladimir@math.ias.edu \\ Charles Weibel (Managing Editor) \\ Rutgers University, USA \\ weibel@math.rutgers.edu \\ Guoliang Yu Texas A\&M University, USA \\ guoliangyu@math.tamu.edu \\ PRODUCTION \\ $\begin{array}{ll}\text { Silvio Levy } & \text { (Scientific Editor) } \\ \text { production@msp.org }\end{array}$
}

Annals of K-Theory is a journal of the K-Theory Foundation (ktheoryfoundation.org). The K-Theory Foundation acknowledges the precious support of Foundation Compositio Mathematica, whose help has been instrumental in the launch of the Annals of K-Theory.

See inside back cover or msp.org/ant for submission instructions.

The subscription price for 2016 is US $\$ 400 /$ year for the electronic version, and $\$ 450 /$ year (+\$25, if shipping outside the US) for print and electronic. Subscriptions, requests for back issues and changes of subscribers address should be sent to MSP.

Annals of K-Theory (ISSN 2379-1681 electronic, 2379-1683 printed) at Mathematical Sciences Publishers, 798 Evans Hall \#3840, c/o University of California, Berkeley, CA 94720-3840 is published continuously online. Periodical rate postage paid at Berkeley, CA 94704, and additional mailing offices.

AKT peer review and production are managed by EditFlow ${ }^{\circledR}$ from MSP.

PUBLISHED BY

mathematical sciences publishers nonprofit scientific publishing

http://msp.org/

(C) 2016 Mathematical Sciences Publishers 


\section{ANNALS OF K-THEORY}

2016

no. 3

Multiplicative differential algebraic $K$-theory and applications

Ulrich Bunke and Georg Tamme

The Balmer spectrum of a tame stack

Jack Hall

Algebraic Kasparov K-theory, II

Grigory Garkusha

The local symbol complex of a reciprocity functor

Evangelia Gazaki 